\title{
Probabilistic assessment of API casing strength in serviceability limit state
}

\author{
L. P. Gouveia ${ }^{1}$ - E. T. Lima Junior ${ }^{1}$ (I) J. P. L. Santos ${ }^{1}$ - W. W. M. Lira ${ }^{1}$ J. L. R. Anjos ${ }^{2}$ F. L. Oliveira ${ }^{2}$
}

Received: 7 September 2019 / Accepted: 17 February 2020 / Published online: 26 February 2020

(c) The Author(s) 2020

\begin{abstract}
This paper addresses the probabilistic analysis of casing tubulars, regarding the failure modes defined in API 5C3 code, which refers to the violation of elastic regime due to internal and external pressures, and axial force. The casing system performs important structural and isolation functions, ensuring the well integrity through its life cycle. The reliability-based casing design handles rigorously the uncertainties associated with the tube manufacturing, as variations in geometrical and mechanical properties, allowing to evaluate the probability of failure. It is presented a parametric analysis over different steel grades and tube slenderness, besides the application to a design scenario, by using Monte Carlo simulation and firstorder reliability method. The results indicate that: collapse is the dominant failure mode; wall thickness and the yield limit govern the probabilistic response; the triaxial envelopes, revisited in a probabilistic framework, consist in a powerful tool, supporting the decision-making process in casing design.
\end{abstract}

Keywords OCTG $\cdot$ Structural reliability $\cdot$ Probabilistic design $\cdot$ Burst $\cdot$ Collapse $\cdot$ von Mises envelope

\section{Introduction}

Casing systems in oil and gas wells play an important role as the major structural system that keeps the well integrity since drilling and along its lifetime. The main elements in this system are tubulars and connections, also referred by OCTG (Oil Country Tubular Goods), which must be designed in order to support the external loadings that they are subjected. In offshore well construction, which includes drilling, casing, cementing and completion stages, the casing system may represent around $18 \%$ of the total cost (Rahman and Chilingarian 1995). The search for oil and gas in increasingly higher depths exposes the casing to extreme conditions, including high levels of pressure and temperature (HPHT), besides chemical attack as, for instance, corrosion due to hydrogen sulfide gas. In this scenario, the adequate balance between cost and safety levels has to be reached in

E. T. Lima Junior

limajunior@1ccv.ufal.br

1 Laboratory of Scientific Computing and Visualization, Federal University of Alagoas, Maceió, Brazil

2 Petrobras, Rio de Janeiro, Brazil the design, and the structural reliability theory can assist the designer in this decision-making process.

The main loadings experienced by tubulars are represented by internal pressure, external pressure and axial force. The failure modes associated with internal and external pressure, so-called burst and collapse, respectively, are the governing failure modes in most cases. Tensile or compressive forces occur along the casing, but normally not induce tube failure themselves. A triaxial analysis can be done, by using an equivalent stress state, as the one proposed by von Mises (1913), in order to verify the combined effects of pressures and axial load. Additional torsion and bending effects, not assessed in this work, can stand out in some scenarios. Mohammed et al. (2019) provide an overview on the structural integrity aspects and failure modes of casing systems, in different well types.

The OCTG strength equations are posed in the code API 5C3 (1999), which is widely applied by oil and gas companies. This formulation refers to a serviceability limit state (SLS) related to the elastic regime. Therefore, the tubes are designed to withstand loads up to reaching the steel yielding limit, disregarding its bearing capacity after this point. This is one of the reasons that makes this code seems conservative, underestimating the actual strength of the tube. The API 5C3 (1999) normative code suggests the Barlow's 
equation for burst design, whose derivation is done by assuming thin wall hypothesis, which can be inadequate in some tubes commonly adopted in casing design. In terms of external pressure, the code provides four collapse pressure formulas for design, developed on the 1960s, being each formula suitable for a distinct $D / t$ interval, where $D$ is the tube outside diameter and $t$ is the wall thickness.

Some authors address the recommended formulas from API 5C3 (1999) and discuss its apparent conservative nature, besides proposing ultimate limit state (ULS) formulations. Some works can be found in the literature with distinct ultimate limit state equation suggestions for burst, as Klever and Stewart (1998) and Klever (2010). ULS modeling for collapse pressure is addressed by Abbassian and Parfitt (1995), Klever and Tamano (2006) and Huang and Gao (2015).

A new version of the standard (API/TR 5C3 2008), identical to ISO 10400 (2007), introduces the ULS philosophy in casing design and suggests that reliability-based procedures can be performed in order to enhance the strength evaluation. An ULS formulation is usually related with experimental test results, since it tries to estimate the load at which the casing tubular fails. More specifically, in this version of the code it remains the collapse formulation, but is also suggested, in an informative annex, a new ULS model based on the Klever and Tamano (2006) work. Regarding the internal pressure, the 2008 version presents a ductile rupture formula based on (Klever and Stewart 1998) replacing the classical Barlow's equation. These new formulations also consider other parameters that affect casing strength like ovality, eccentricity, residual stresses and ultimate tensile strength. Although these proposed improvements, the 1999 former models are still widely applied in design routines, being the model adopted in most wells in service. The new paradigm of ULS associated with probabilistic design is slowly being introduced in the companies by consulting and research activities.

The probabilistic approach aims to the accounting of uncertainties in the structural analysis, to estimate the failure probability of a structural element or system. The reliability theory allows this estimation, for specified limit states, by the statistical description of the design variables. Melchers and Beck (2017) and Ditlevsen and Madsen (2005) present the fundamentals of structural reliability.

Specifically, in oil and gas industry, well casing design is related to steel tubular manufacturing, whose production quality and inspection procedures have been improved significantly in the last decades. The suggested SLS strength formulas are dependent on the outside diameter $(D)$ and wall thickness $(t$ ) - usually referred by the slenderness ratio $D / t$ - and yield stress. In this case, the former version of API code recommends adopting conservative minimal or nominal values for these parameters and, additionally, in the design process, safety factors are applied to implicitly ensure a tolerable risk level
(TRL). On the other hand, if the supplier guarantees accuracy in the manufacture and inspection processes, with lower dispersion levels in the tube performance values, consequently the TRL can be kept by allowing the structural element to bear a higher load than the one predicted by the standard.

Since the 1990s, some authors have applied probabilistic design theory to OCTG. Burres et al. (1998) present an interesting discussion working on the calibration of design safety factors in order to reach a specific TRL. Adams et al. (1998) present the behavior of failure probabilities for a ULS collapse strength formulation proposed by Tamano et al. (1985) and verify that it gives a wide range of variation for failure probabilities over a $D / t$ range, concluding that this behavior is not desirable and suggesting a new reliability-based method for collapse casing design. (Ju 1998) proposes a different ULS formulation, based on an experimental database of collapse tests.

The code API/TR 5C3 (2008) itself does a very similar development, in adopting the reliability-based calibration of two down-rating factors present in the Klever and Tamano (2006) model. Liao et al. (2012) apply Monte Carlo simulation to obtain casing failure probability, based on both Klever-Stewart and Klever-Tamano models. Gao et al. (2019) address the probabilistic response of casing strings subject to seismic actions, by using spectral modal analysis. It is noticed that the papers dealing with probabilistic casing analysis mostly employ the Monte Carlo simulation, which can become unfeasible for design practice purposes, due to the high computational cost associated.

This work deals with the classical SLS formulation, considering this is still the most representative of the wells in service, despite the recent ULS models published. The first-order reliability method (FORM) and the Monte Carlo simulation are used to perform the probabilistic analysis. The evolution of safety levels associated with the referred equations across $D / t$ and for distinct grades is investigated, and a performance comparison between these methods is carried out. Moreover, a typical design scenario is evaluated by using the proposed methodology, in order to illustrate its application in the casing design practice.

The paper is divided in five main sections. The second one has a brief review on the structural reliability analysis. The third one brings an overview of the recommended practices for casing design in SLS regime. In fourth section, the methodology and examples are presented, discussing the results. Finally, in the last section, some concluding remarks are presented.

\section{Structural reliability analysis}

A structural reliability analysis comprises a limit state function, random variables (r.v.) and a reliability evaluation method. The limit state function represents the 
physics of the problem under study, being arranged to provide negative values for failure events. The failure modes addressed in this paper represent the safety margin of probabilistic API 5C3 casing strength be exceeded by a deterministic load. In Eq. 1 is defined the failure function $G(\boldsymbol{X})$, where $R(\boldsymbol{X})$ is the strength term and $L$ is the load term, as follows:

$G(X)=R(X)-L$

where the vector $\boldsymbol{X}$ holds the adopted random variables. In the present analysis, only the resistance term is described as probabilistic. The random variables should describe the geometric and mechanical parameters related to the structure, which influence the resistance term in the failure function. In a more general approach, the load term $L$ also could have r.v., as the self-weight, fluid density, pore pressure, temperature variation and others.

The reliability methods evaluate the limit state function taking into account the randomness of the design variables, to compute a failure probability. For structural reliability, the Monte Carlo simulation and FORM are the most known ones (Melchers and Beck 2017). In the next sections, these methods are briefly described.

\section{Monte Carlo simulation}

The simulation is carried out by generating $n$ random scenarios to be verified in the limit state function, computing the number of failures $n_{f}$ (when $G(\boldsymbol{X}) \leq 0$ ), and evaluating the failure probability by the relation $P_{f}=n_{f} / n$. An illustrative case of a Monte Carlo simulation is presented in Fig. 1, in which several events are generated to the analysis of the failure function $G(R, L)=R-L$, whose variables $R$ and $L$ are taken as Gaussian.

The results provided by the Monte Carlo method tend to be very accurate, if an adequate number of simulations is performed. However, the computational cost increases linearly with the number of simulations required, becoming prohibitive in problems presenting low failure probabilities. Some alternative techniques can be applied to improve performance in this method as, for instance, importance sampling procedures, response surface approximations and the usage of polynomial chaos expansion.

Regarding the feasibility of Monte Carlo, at a first sight, it is needed, at least, the inverse of the failure probability number of scenarios to possibly be capable to detect one failure event, i.e., if the problem has a $P_{\mathrm{f}}$ equals to $10^{-6}$, a minimum of $10^{6}$ scenarios must be generated and simulated. However, due to the variability on the generation of random scenarios, at each set of realizations, it follows that the estimated $P_{\mathrm{f}}$ value is also a random variable, and some

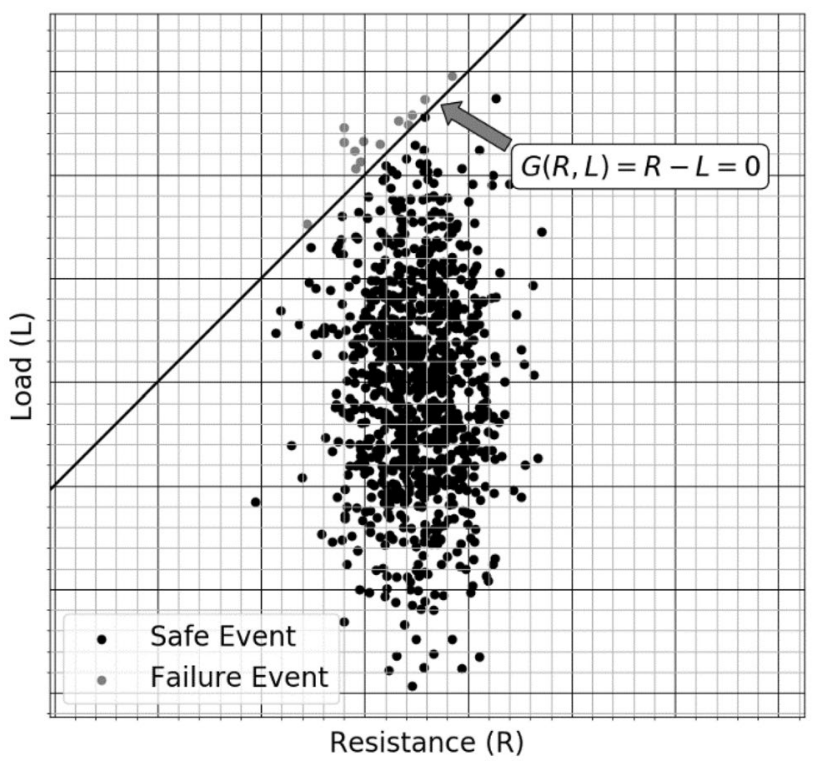

Fig. 1 Monte Carlo illustrative example

expressions are proposed to define a minimum number of scenarios that leads to a desirable coefficient of variation of $P_{\mathrm{f}}\left(\mathrm{COV}_{P f}\right)$, in a single simulation.

This $\mathrm{COV}_{\mathrm{Pf}}$ can be evaluated by $\mathrm{COV}_{\mathrm{Pf}}=\sigma_{P_{f}} / \mu_{P_{f}}$, where $\sigma_{P_{f}}=\sqrt{\sum_{i}^{N}\left(P_{f i}-\mu_{P_{f}}\right) /(n-1)}, \mu_{P_{f}}=\sum_{i}^{n} P_{f i} / n$ and $P_{f i}$ is the failure probability evaluated in scenario $i$. Based on this definition, Ang and Tang (2007) propose the following expression for the minimum number of scenarios, $n_{\min }=\left(1-P_{f}^{\prime}\right) /\left(\operatorname{COV}_{\mathrm{Pf}}^{2} P_{f}^{\prime}\right)$, in which $P_{f}^{\prime}$ is the characteristic $P_{f}$ value of the problem under study.

\section{First-order reliability method}

The reliability problem can be stated by using a limit state function $(G(\boldsymbol{X})=0)$, in terms of the random variables $\boldsymbol{X}$, being $P_{f}$ evaluated as follows:

$P_{f}=P(G(\boldsymbol{X}) \leq 0)=\int_{G(\boldsymbol{X}) \leq 0} f_{\boldsymbol{X}}(\boldsymbol{x}) \mathrm{d} \boldsymbol{x}$

in which $f_{\boldsymbol{X}}(\boldsymbol{x})$ is the joint probability density function (pdf) of the random variables $\boldsymbol{X}$. The solution of this integral may not be trivial, due to the nonlinear nature of the joint pdf and in the case of many r.v. be considered. Discrete procedures can be performed, as the Monte Carlo simulation above mentioned. Other iterative transformation techniques as the first-order reliability method (FORM) stand out as an alternative. The main idea is to transform the random variables $\boldsymbol{X}$ in its standardized normally distributed ones $\boldsymbol{Y}$, by applying the so-called normal tail approximation. The

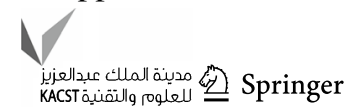


limit state function is also represented in the standard normal space $(G(\boldsymbol{Y})=0)$. As the main premise of the transformation methods, it stands that the shortest distance between the origin of the transformed normal space and the new limit state function is known as the reliability index $\beta$, which can be related with $P_{\mathrm{f}}$ by Eq. 3 .

$P_{f}=\Phi(-\beta)$

The reliability analysis is stated as a nonlinear optimization problem, in which one wants to minimize the distance $\beta$ subject to the constraint function $G(\boldsymbol{Y})=0$. The point $\boldsymbol{Y}^{*}$, socalled design point, contains the most probable values of the r.v. which leads to failure. Thus, the reliability index corresponds to the norm of the position vector of this point, i.e., $\beta=\boldsymbol{Y}^{*}$. The random variables transformation is made by the Normal Tail Approximation, as suggested by Hasofer and Lind (1974). The new limit state function is approximated by a first order Taylor series at the current search point from the iterative optimization procedure. The optimization problem can be stated as in Eq. 4 .

$\beta=\min (\boldsymbol{Y})$, constrained to $G(\boldsymbol{Y})=0$

The Hasofer and Lind (1974), Rackwitz and Fiessler (1978) algorithm (HLRF) is classically employed to the optimization problem solution. In general, the convergence is reached in a few iterations (less than 10), at a computational cost quite inferior to the Monte Carlo one, with a good level of accuracy, in several applications. Moreover, the FORM results are exact for linear failure functions, whose random variables are Gaussian. Figure 2 illustrates the procedure.

By using FORM, it can be calculated the importance factor of each random variable, for the failure probability obtained. This information is related to the position vector $\boldsymbol{Y}^{*}$ and the partial derivatives of $G(\boldsymbol{Y})$ at this point.

It should be noted that, in the case of correlation between random variables, additional steps are necessary. In order to calculate the equivalent correlation coefficient for each pair of variables in the transformed normal space, the procedure proposed in Nataf (1962) can be applied. Moreover, this correlation has to be eliminated, so that the final transformation into standardized normal independent variables $\boldsymbol{Y}$ can be performed. These procedures are detailed in Melchers and Beck (2017).

In some cases, in which the variables are tightly correlated, or present non-Gaussian distributions, or when the limit state function is strongly nonlinear, the use of a second-order approximation of the limit state function can improve the accuracy of results obtained in the transformation method. It gives rise to SORM (second-order reliability method). This approximation demands more information over the limit state function, as its curvatures. The final approximation consists in a parabolic equation centered on the design point. For this quadratic approximation, the algorithm presented by Breitung (1984) can be employed.
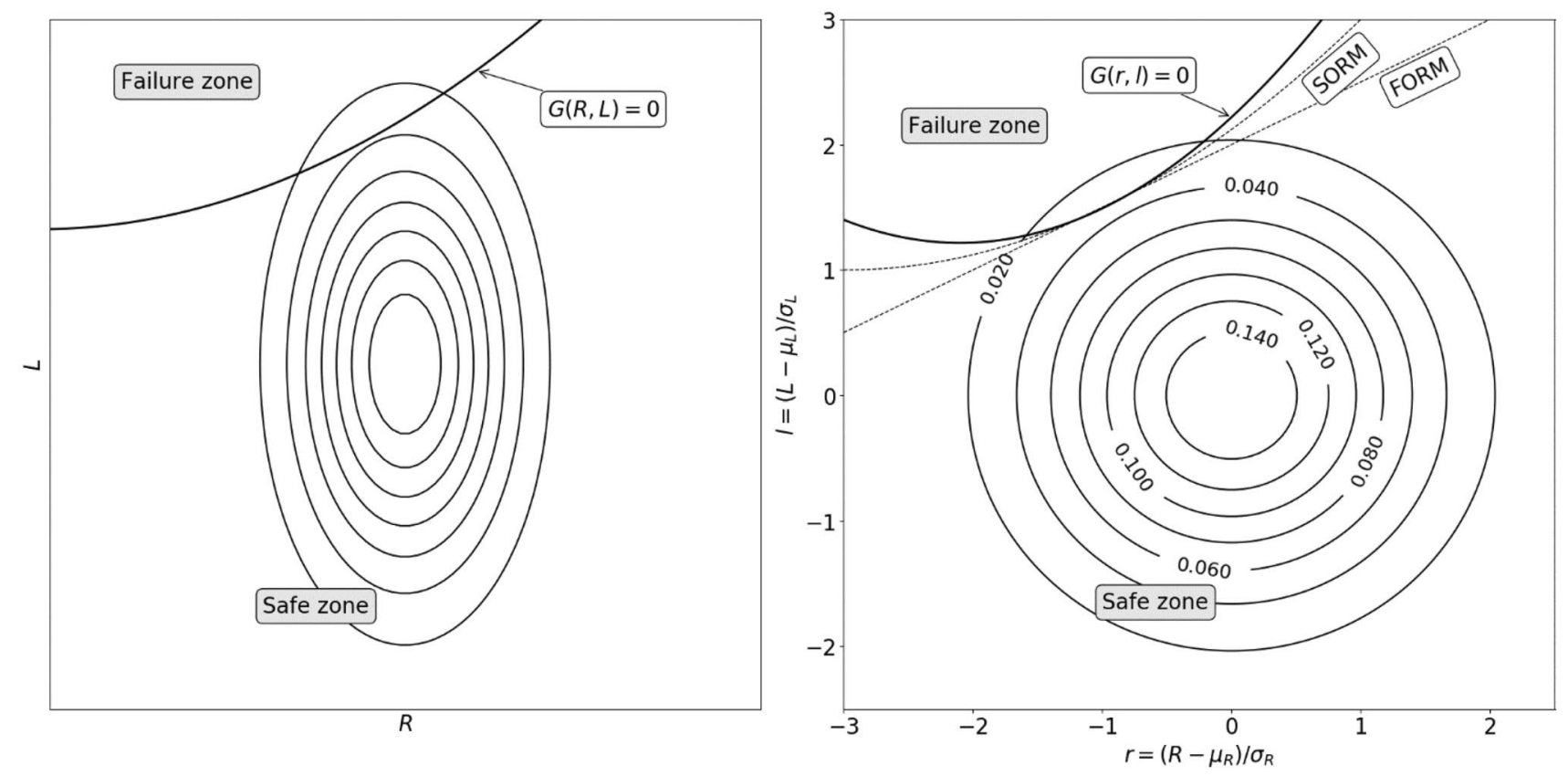

Fig. 2 FORM illustrative Scheme: original and standard normal spaces 


\section{Casing strength formulation}

The recommended practice for well casing design described by API 5C3 (1999) is summarized in this section, focusing the axial, burst and collapse strength.

\section{Burst strength}

The internal pressure that leads to a stress, on the inside wall, equals to the minimum yield strength. The failure mode associated is the burst on the tube. The equation is based on the Barlow's equation, suitable to thin wall tubes:

$P_{\mathrm{b}}=0.875\left(\frac{2 Y_{p} t}{D}\right)$

where $D$ is the outside diameter, $t$ is wall thickness, and $Y_{p}$ is the minimum steel yielding limit. The reduction factor 0.875 refers to a tolerance of $-12.5 \%$ in the wall thickness. This value is the allowable limit due to non-uniformity in manufacture process and is defined by the code API 5CT (2010). This is one of the reasons why the equation seems to be conservative.In the presence of axial force, no corrections are applied to burst strength formulation. In the presence of external pressure $P_{e}$, the strength value is compared with differential pressure $\Delta P=P_{i}-P_{e}$.

This classical Barlow's model is revisited in Adams et al. (2018), in which the assumptions and limitations are carefully described, in order to discuss its validity against the combined von Mises model and modern ductile rupture models.

\section{Collapse strength}

When a pipe collapses due to external load, it changes the geometry to elliptical or other non-circular shape. It brings structural problems associated with loss of stiffness and local instability, besides operational issues as blocking of passage of equipment into the tube. The external load is usually caused by pore pressure, pressure from the backup drilling fluid, cement slurry, or annular pressure build-up (APB). According to API 5C3 (1999), four distinct casing slenderness $D / t$ domains compose the collapse design strength. Yield strength collapse pressure formula (Eq. 6) provides the load that generates minimum yield stress $Y_{p}$ on the inside wall of the tube. This formula is achieved by means of Lamé classical equation.

$P_{\mathrm{cy}}=2 Y_{p}\left[\frac{(D / t)-1}{(D / t)^{2}}\right]$
Average plastic collapse pressure formula (Eq. 7) was derived empirically from several collapse tests for casing tube grades K55, N80 and P110. This is the usual nomenclature for the steel grade, where the digits refer to its minimum yield stress, in ksi. The data used to fit the equation were taken from a report developed by a workgroup composed by manufacturers and members from API itself. Collapse tests data were fitted separately for each grade, and then, constants $A$ and $B$ were empirically determined to generalize an average plastic collapse pressure formula. In order to obtain the minimum plastic collapse pressure formula for a particular grade, a constant $C$ is subtracted from the average expression. This constant $C$ is a tolerance limit and represents the hypothesis that there is a $95 \%$ probability or confidence level that the collapse pressure will exceed the minimum stated with no more than $0.5 \%$ failures.

$P_{c p}=Y_{p}\left[\frac{A}{(D / t)}-B\right]-C$

Transition collapse pressure formula (Eq. 8) overcomes an anomaly that happens between minimum plastic collapse formula and minimum elastic collapse formula: they do not intersect across the $D / t$ range. Thus, this formula has been developed intersecting the $D / t$ value where the average plastic collapse pressure formula (i.e., Equation 8 without $\mathrm{C}$ term) gives a collapse pressure of zero and is tangent to the minimum elastic collapse pressure.

$P_{\mathrm{ct}}=Y_{p}\left[\frac{F}{(D / t)}-G\right]$

Finally, the minimum elastic collapse pressure formula (Eq. 9) was derived from theoretical elastic collapse theory, being applied penalizations of $95 \%$ and $75 \%$.

$P_{\mathrm{ce}}=\frac{46.95 \times 10^{6}}{(D / t) \times((D / t)-1)^{2}}$

The $D / t$ limits which define the collapse domains, defined by the intersection of the collapse pressure formulas described above, are presented in Eqs. 10-12.

$(D / t)_{\mathrm{Yp}}=\frac{\sqrt{(A-2)^{2}+8\left[B+\left(\frac{C}{Y_{p}}\right)\right]}+(A-2)}{2\left[B+\left(\frac{C}{Y_{p}}\right)\right]}$

$(D / t)_{\mathrm{PT}}=\frac{Y_{p}(A-F)}{C+Y_{p}(B-G)}$ 
$(D / t)_{\mathrm{TE}}=\frac{2+B / A}{3 B / A}$

These $D / t$ limits are dependent only on the yield stress (in psi) and must be evaluated for each steel grade. Once they are determined, it is necessary to verify the casing design collapse domain by the tube slenderness $D / t$. If casing $D / t$ is lower than $(D / t)_{\mathrm{Yp}}$, yield collapse pressure formula must be applied. If casing $D / t$ is higher than $(D / t)_{\mathrm{Yp}}$, but lower than $(D / t)_{\mathrm{PT}}$, minimum plastic collapse pressure formula is adequate. If casing $D / t$ is higher than $(D / t)_{\mathrm{PT}}$, but lower than $(D / t)_{T E}$, transition collapse pressure formula must be applied. If casing $D / t$ is higher than $(D / t)_{\mathrm{TE}}$, minimum elastic collapse pressure formula must be considered. The coefficients $A, B, C, F$ and $G$ are shown below.

$$
\begin{aligned}
A= & 2.8762+0.10679 \times 10^{-5} Y_{p} \\
& +0.21301 \times 10^{-10} Y_{p}^{2}-0.53132 \times 10^{-16} Y_{p}^{3}
\end{aligned}
$$

$B=0.026233+0.50609 \times 10^{-6} Y_{p}$

$C=-465.93+0.030867 Y_{p}-0.10483 \times 10^{-7} Y_{p}^{2}+0.36989 \times 10^{-13} Y_{p}^{3}$

$$
F=\frac{46.95 \times 10^{6}\left[\frac{3(B / A)}{2+(B / A)}\right]^{3}}{Y_{p}\left[\frac{3(B / A)}{2+(B / A)}-(B / A)\right]\left[1-\frac{3(B / A)}{2+(B / A)}\right]^{2}}
$$

$G=\frac{F B}{A}$

The collapse strength of casing in the presence of an axial stress is calculated by modifying the yield stress to an equivalent value, according to:

$Y_{\mathrm{pa}}=\left[\sqrt{1-0.75\left(S_{\mathrm{a}} / Y_{p}\right)^{2}}-0.5\left(S_{\mathrm{a}} / Y_{p}\right)\right] Y_{p}$

where $S_{\mathrm{a}}$ is the axial stress, $Y_{p}$ is the minimum yield strength, and $Y_{\mathrm{pa}}$ is the yield strength of axial stress equivalent grade, in psi. The new value of yield stress $Y_{p a}$ must be applied in all formulation, including in the definition of $D / t$ limits (Eqs. 10-12). When $Y_{p a}$ is lower than 24,000 psi, the formulation is not valid and cannot be applied.

In presence of internal pressure $P_{i}$, the collapse strength of the tube is compared with equivalent differential pressure, given by Eq. 14.

$\Delta P_{e}=P_{e}-(1-2 /(D / t)) P_{i}$

\section{Axial strength}

The axial force $\left(R_{t}\right)$ that corresponds to a stress equal to the minimum yield strength is given as follows:

$R_{t}=0.7854\left(D^{2}-d^{2}\right) Y_{p}$

where $d$ is the specified inside diameter. The axial force is the result of the balance between self-weight of the tube and the buoyancy caused by the drilling fluid and other fluids from formation, and it does not configure a governing failure mode by itself, as it will be presented in "Analysis and results" section.

\section{Combined loads design}

The mechanical model used to represent this limit state consists of von Mises criterion combined with Lamé equations evaluated on the casing inner radius. Accordingly to API/ TR 5C3 (2008), the onset of yielding in a casing tube will not occur if $\sigma_{e}<Y_{p}$ in which the von Mises equivalent stress $\sigma_{e}$ is given by

$\sigma_{e}=\sqrt{\sigma_{r}^{2}+\sigma_{h}^{2}+\sigma_{a}^{2}-\sigma_{r} \sigma_{h}-\sigma_{r} \sigma_{a}-\sigma_{h} \sigma_{a}}$

where $\sigma_{r}$ is the radial stress, $\sigma_{h}$ is the hoop stress, and $\sigma_{a}$ is the axial stress. Design scenarios are usually given by loads of combined axial force $F_{a}$, internal pressure $P_{i}$ and external pressure $P_{e}$ acting on casing tube. Then, $\sigma_{a}$ is given by

$\sigma_{a}=\frac{F_{a}}{\pi t(D-t)}$

in which the denominator is equivalent to the tube crosssectional area. Lamé equations for thick tubes applied on inner radius are written in the form

$\sigma_{r}=-P_{i}$

$\sigma_{h}=-P_{i}+\Delta P \frac{D^{2}}{2 t(D-t)}$

where $\Delta P$ is the differential pressure given by $P_{i}-P_{e}$.This formulation refers to inner radius stress values, where occurs the maximum critical levels if torsional or bending stresses are not considered.

Usually, the combined loads evaluation is also visualized in an envelope graph where loads are quickly compared with tube strengths. Classical von Mises envelope plotted in a Cartesian space with $\Delta P$ in y-axis and $F_{a}$ in x-axis is a simplified version of formulation presented above. In summary, it is considered that when $\Delta P>0$ the external pressure is zero, and when $\Delta P<0$ the internal pressure is zero. 

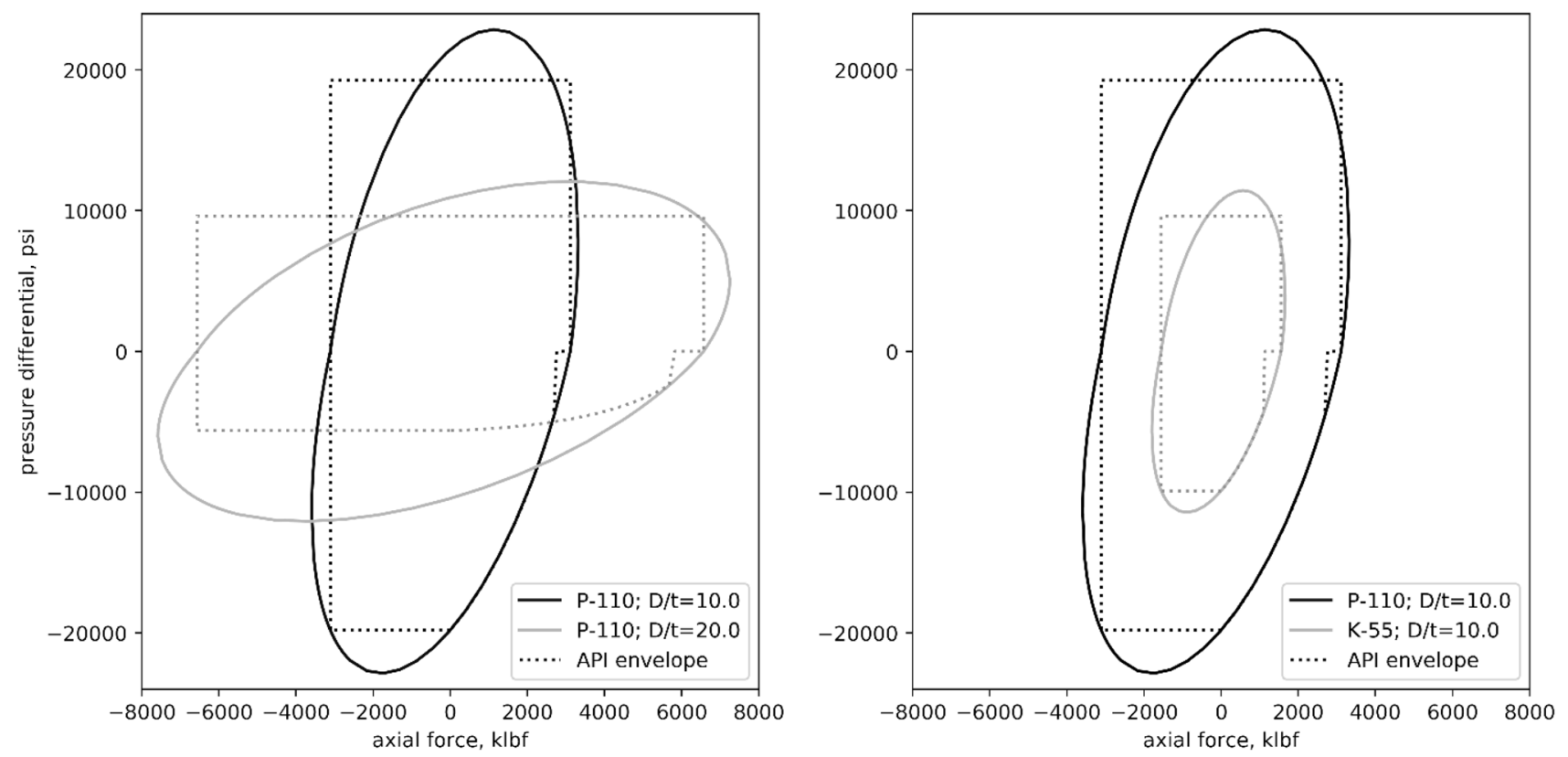

Fig. 3 von Mises ellipse and API envelope for some tube configurations, varying $\boldsymbol{D} / \boldsymbol{t}$ and $\boldsymbol{Y}_{\boldsymbol{p}}$ values

This simplification leads to a distorted ellipse presented in Fig. 3, varying $D / t$ and $Y_{p}$. It is observed that increasing $D / t$ induces the ellipse to rotate clockwise and increasing $Y_{p}$ the ellipse tends to expand.

The envelope delimited by the safety factors provided in API code is also plotted in Fig. 3. It can be pointed out, for example, that for $D / t=10.0$ a thick-walled tube presents an API collapse strength congruent to the von Mises ellipse, as verified in the lower portion of the figure. For thin tubes, as the one presented $(D / t=20.0)$, the API envelope is more conservative for the collapse strength, compared to the von Mises criteria. In casing design routines, both envelopes are considered in decision-making process. The selection of an adequate tube configuration consists in verifying if the estimated load points are contained in both envelopes.

\section{Analysis and results}

In order to address the concepts presented in previous sections, two kinds of reliability analysis are performed, as follows. The first one is done by verifying the probability of API 5C3 (1999) design strength $\left(R_{\text {prob }}\right)$, in which the r.v. are considered, be exceeded by a solicitation equal to its deterministic value $\left(R_{\text {det }}\right)$, i.e., the design strength value. In this case, both single pressure and combined pressures analysis are carried out. The second one is a practical evaluation of the probability of API 5C3 (1999) design limits be exceeded in a usual load scenario.
In the first analysis, presented in "Collapse, burst and axial design formulation analyses" and "Combined loads design formulation analysis" sections, the load is going to be adopted to the maximum value that current deterministic design methodologies permits. This kind of analysis can be useful to verify the probability of this design strength be exceeded, leading the casing tube to transcend the elastic limit. Also, it is possible to evaluate the influence of the dispersion of variables in each strength model, across the $D / t$ range. In this manner, the following investigations are carried out:

a. Burst strength without external pressure and axial force;

b. Collapse strength without internal pressure and axial force;

c. Axial strength;

d. Triaxial strength (von Mises) with combined internal/ external pressure, and axial force.

The second analysis ("Typical Design Scenario" section) refers to a common design scenario with its load profiles, for which the failure probability is evaluated along the well depth. This application becomes useful when the results are compared to the safety factors obtained from the deterministic design. The main objective of this analysis consists in present a methodology for supporting casing design decision-making process with probabilistic information of tubular strength.

In this work, the term failure probability indicates the probability of exceeding the serviceability limit state usually adopted in industry and recommended by API code. Thus, the results consist mainly on the probabilities of casing tubes

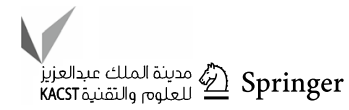


initiate to yield on its inner radius. An exception occurs in thin tubes under collapse loads, where buckling instability of cross section is the failure mode predicted by API code.

The set of random variables contains the yield strength $Y_{p}$, the outer diameter $D$ and the wall thickness $t$, and its statistical parameters are taken from the code ISO 10400 (2007). The referred statistical database compiles several manufacturing production data, between 1977 and 2004, being representative of different manufacturing technologies and quality levels. The statistical parameters are evaluated by using the coefficients shown in Table 1 , for the three steel grades adopted, K55, N80 and P110. The grades represent that the steel used has a minimum yield strength of 55,000 psi, 80,000 psi and 110,000 psi, respectively. In this table, mean is equal to the actual mean value divided by the nominal value, and COV is the standard deviation divided by the actual mean value. According to the reference, the variables are normally distributed.

The admissible values for the failure probability are not well established in the structural engineering community. It depends, for instance, on the class of the structure, and to the costs associated with the failure mitigation and occurrence. The risk of human losses and environmental damages is also determinant aspects on the definition of a tolerable

Table 1 Statistical parameters adopted for the random variables

\begin{tabular}{clllll}
\hline$Y_{p}$ & & $D$ & & $t$ & \\
\hline Mean & COV & Mean & COV & Mean & COV \\
K55 & & 1.0059 & 0.00181 & 1.0069 & 0.0259 \\
1.23 & 0.0719 & & & & \\
N80 & & & & & \\
1.21 & 0.0405 & & & & \\
P110 & & & & & \\
1.10 & 0.0360 & & & & \\
\hline
\end{tabular}

probability of failure. Some normative codes just begin to define reference values, e.g., the Joint Committee on Structural Safety (JCSS 2002). In well design industry, it consists in a subject of relatively incipient discussion. Probabilities of failure higher than $10^{-3}$ are treated here as concerning values.

\section{Collapse, burst and axial design formulation analyses}

The probabilistic strength formula is obtained by adopting the variables as random in the API 5C3 design equation. The deterministic load is set equals to the value of API 5C3 design strength, calculated on the nominal values. It means that the failure probability achieved represents the probability of the design strength be exceeded if there is a load equal to the minimum strength currently used in design. Thus, the limit state function can be written as:

$G\left(Y_{p}, D, t\right)=R_{\text {prob }}\left(Y_{p}, D, t\right)-R_{\mathrm{det}}$

Figure 4 presents the results for collapse reliability analysis without internal pressure and axial force.

The four collapse domains described in the previous section present different failure probabilities over $D / t$ range, besides the theoretically derived formulas give nearly constant failure probabilities (yield and elastic collapse pressure formulas), and the empirical formulas give variable failure probabilities (plastic and transition collapse pressure formulas). For the three grades, it is noticed that the probability of failure grows as the parameter $D / t$ increases, reaching inadmissible values superior to $10^{-1.0}$, being more pronounced in the elastic collapse regime (Eq. 9). In this case, the randomness considered in the resistance term of Eq. 20 is only due to the r.v. associated with $D$ and $t$, so that the difference between the terms $R_{\text {prob }}$ and $R_{\text {det }}$ becomes small, which translates in high $P_{f}$ values. The discontinuity
Fig. 4 Collapse failure probabilities over $\mathrm{D} / \mathrm{t}$ range

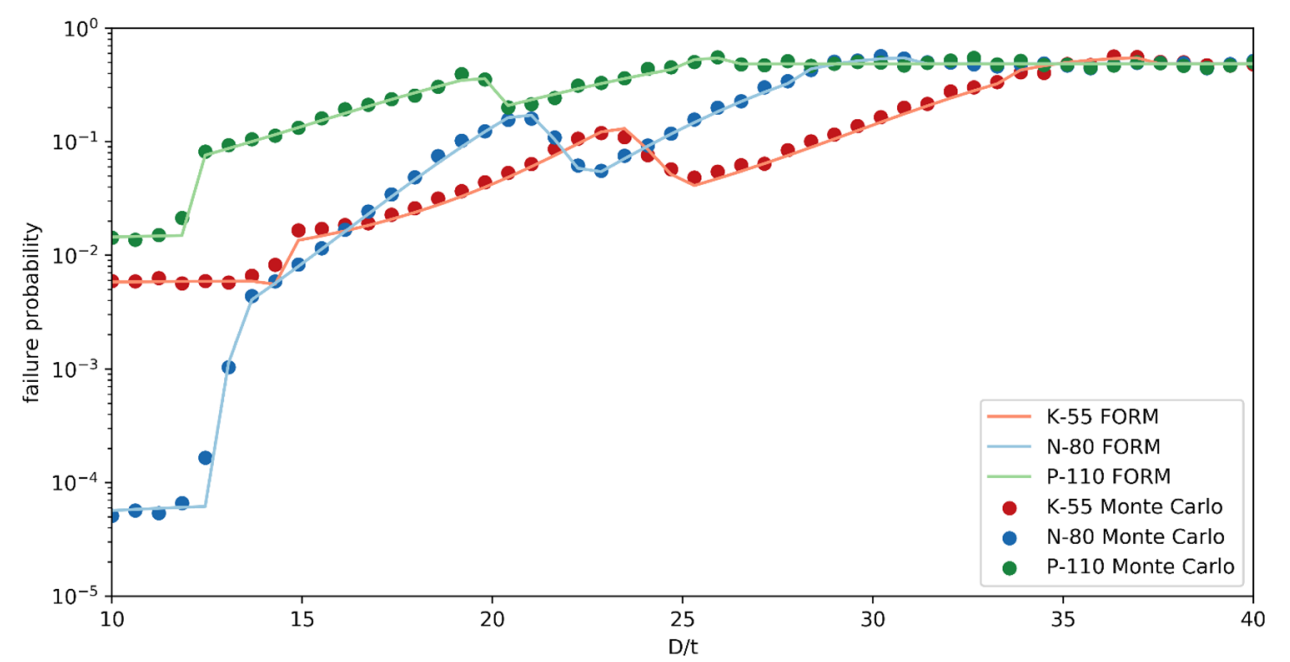


of $P_{f}$ values between different regimes is also verified and can be associated with the four different equations, sharply interconnected. This kind of behavior is not desirable in a design formulation, since it is expected constant safety level for all casing tubes designed by the same normative code. This is one of the main arguments posed by the committee which worked on the new version of the code (API/TR 5C3 (2008)), proposing only one ULS formulation, and encouraging the uses of probabilistic analysis in design.

It must be noted that the correction factor 0.875 is not used in the term $R_{\text {prob }}\left(Y_{p}, D, t\right)$ in burst analysis. The variability of wall thickness is consistently addressed here, by assuming it as a random variable. The analysis of burst formula is presented in Fig. 5.

In this case, the failure probabilities have a constant behavior over the $D / t$ range. However, the failure probabilities are not constant when the casing grade varies, assuming values lower than $10^{-4.0}$. It is noticed that grade N80 has a much lower failure probability than the other two grades, increasing significantly the required number of scenarios, exceeding the computer memory, which is why the Monte Carlo results are missing. This behavior occurs because mean and COV of $Y_{p}$ vary with grade. However, the design formulation should have been calibrated to achieve a target reliability level considering the statistical data from production.

Regarding the axial strength model, the terms $R_{\text {prob }}\left(Y_{p}, D, t\right)$ and $R_{\text {det }}$ are evaluated by using Eq. 15 , and the results are presented in Fig. 6 . The values of $P_{\mathrm{f}}$ do not vary with the tube slenderness, oscillating from $10^{-4.7}$ to $10^{-2.1}$, the latter being associated with P110 grade, whose yielding limit presents the lowest $\mathrm{COV}$, but also the lowest mean value, which implies in its reduced performance.

FORM results agree with Monte Carlo in the three reliability analyses presented. The nature of equations and its smooth nonlinearity contributes to this fact. Graphical visualization demonstrates a good agreement between Monte Carlo and FORM, for $P_{\mathrm{f}}$ values greater than $\sim 10^{-4.4}$. The
Fig. 5 Burst failure probabilities over $\mathrm{D} / \mathrm{t}$ range

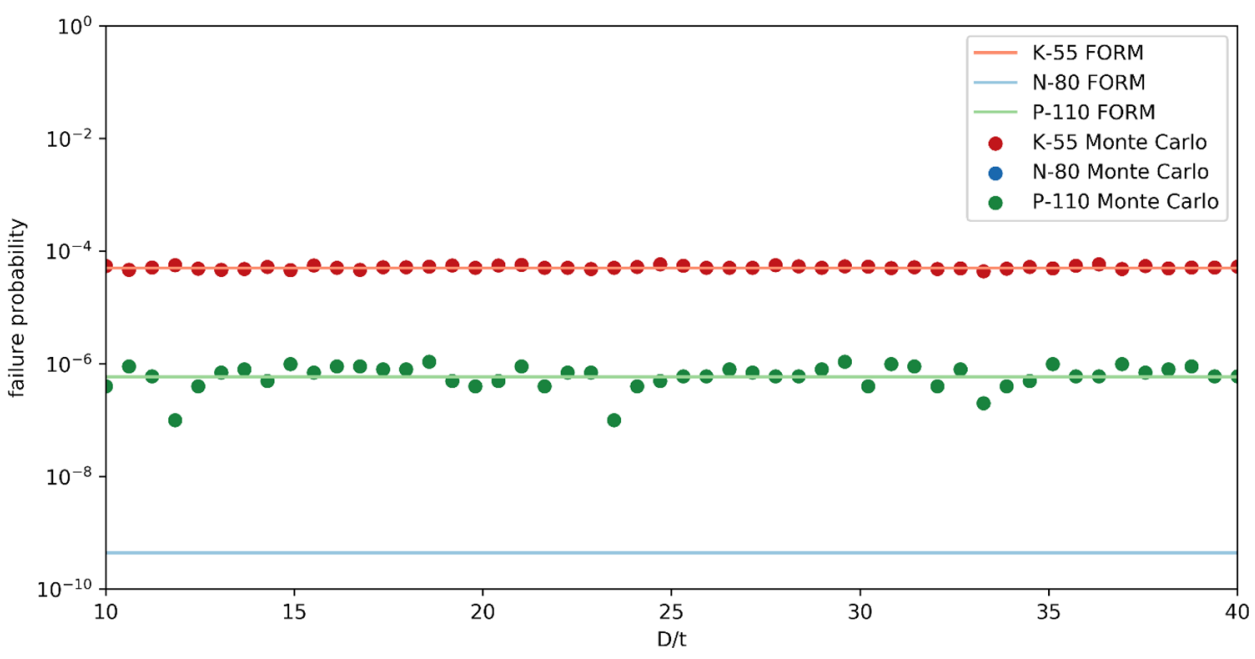

Fig. 6 Axial failure probabilities over $\mathrm{D} / \mathrm{t}$ range

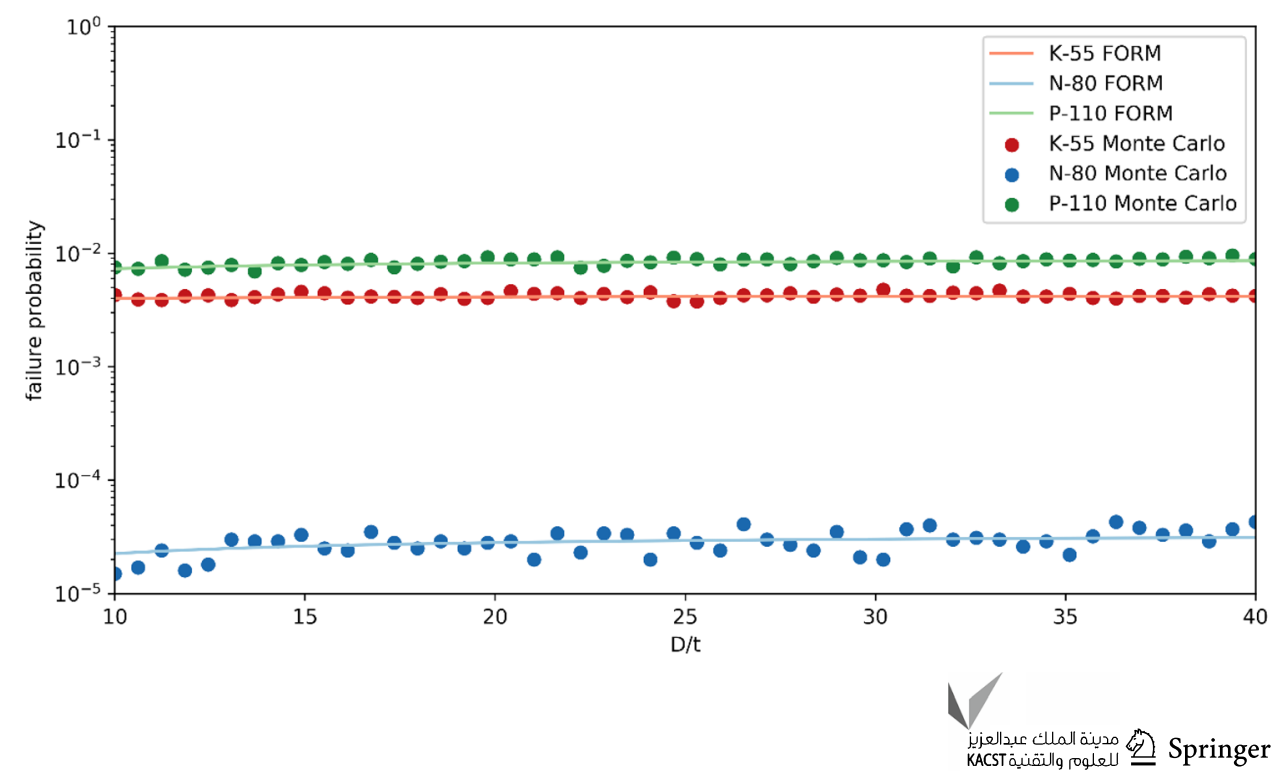


number of Monte Carlo simulations varies depending of the expected $P_{f}$ to be achieved and the admissible dispersion of these $P_{\mathrm{f}}$ results. Considering this, a coefficient of variation, i.e., an admissible error, of $5 \%$ for $P_{\mathrm{f}}$ is set and the number of scenarios is calculated. As $P_{f}$ reduces the number of scenarios and the dispersion of results increase. As an example, in Fig. 6, grade N-80 has $P_{\mathrm{f}}$ around $10^{-5}$ and the number of simulations exceeds $10^{7}$; consequently, the admissible error is not kept in $5 \%$ and the results are visually more dispersed.

The importance factors (IF) obtained by FORM technique for collapse analysis are shown in the following Fig. 7, in which the yield limit is identified by [fy]; the diameter is represented by [od] and the wall thickness by [wt].

Regarding the results presented, the most influent random variable in the process is the wall thickness, except for thick casing tubes in which the yield stress governs the probabilistic behavior. On the other hand, the diameter has a negligible influence in all these results. It means that considering it as deterministic will not affect significantly the failure probability values. These importance factors results are mainly impacted by the formulation used and by the adopted dispersion for each random variable, as it can be noticed in Table 1 that the diameter has the lowest COV.

FORM burst analysis provides the importance factors shown in Fig. 8. The yield stress is the most influent random variable in the achieved failure probability, followed by the wall thickness in all grades. Observing Table 1 and Fig. 7, it can be seen that the higher $C O V$ induces the higher importance factor for K55 grade. For the other grades, it is noticed the decrease in the influence of $Y_{p}$ for N80 and
P110, respectively, but still contributing to $70 \%$, at minimum, of the $P_{\mathrm{f}}$ values. Once again, external diameter is the less important random variable, meaning that its dispersion is very small.

The results obtained for the axial load failure mode (Fig. 9) indicate that the yield limit is the dominant variable, presenting importance factors superior to $90 \%$ in the cases evaluated. For all grades, there is a slightly reduction in its influence as the $D / t$ increases, in which the influence of wall thickness takes place. From grades K55 to P110, in this order, the COV of $Y_{p}$ decreases (see Table 1), so the IF associated with wall thickness ranges from 70 to $92 \%$.

\section{Combined loads design formulation analysis}

The reliability analyses presented in this section adopt the same random variables of previous section, but the limit state function considers combined loads acting on casing tubes. This is accomplished by applying internal pressure, external pressure and axial force with values verified in API envelope and von Mises ellipse ("Axial strength" section). In this way, similarly to the previous section, the analyses present the maximum probability of exceeding SLS in design, that happens when the load reaches the resistance envelopes.

Figure 10 presents the probabilities of exceeding SLS through API envelope for two values of $D / t$, and K55, $\mathrm{N} 80$ and P110 grades. The $P_{\mathrm{f}}$ values associated with limit points of burst and collapse are displayed. These results are obtained by FORM, considering the good convergence observed in previous section.
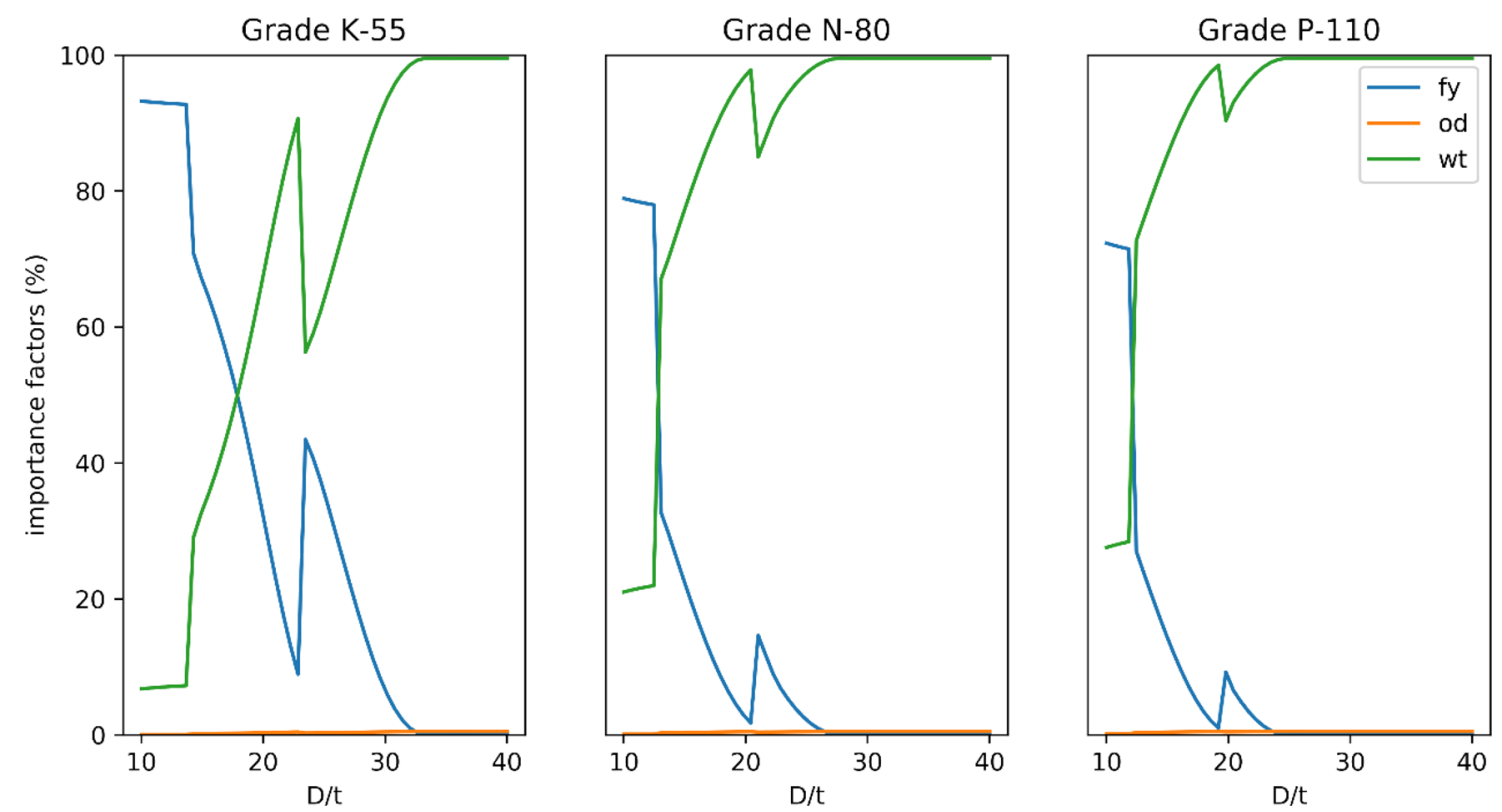

Fig. 7 Importance factors over $\mathrm{D} / \mathrm{t}$ range for collapse analysis

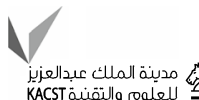



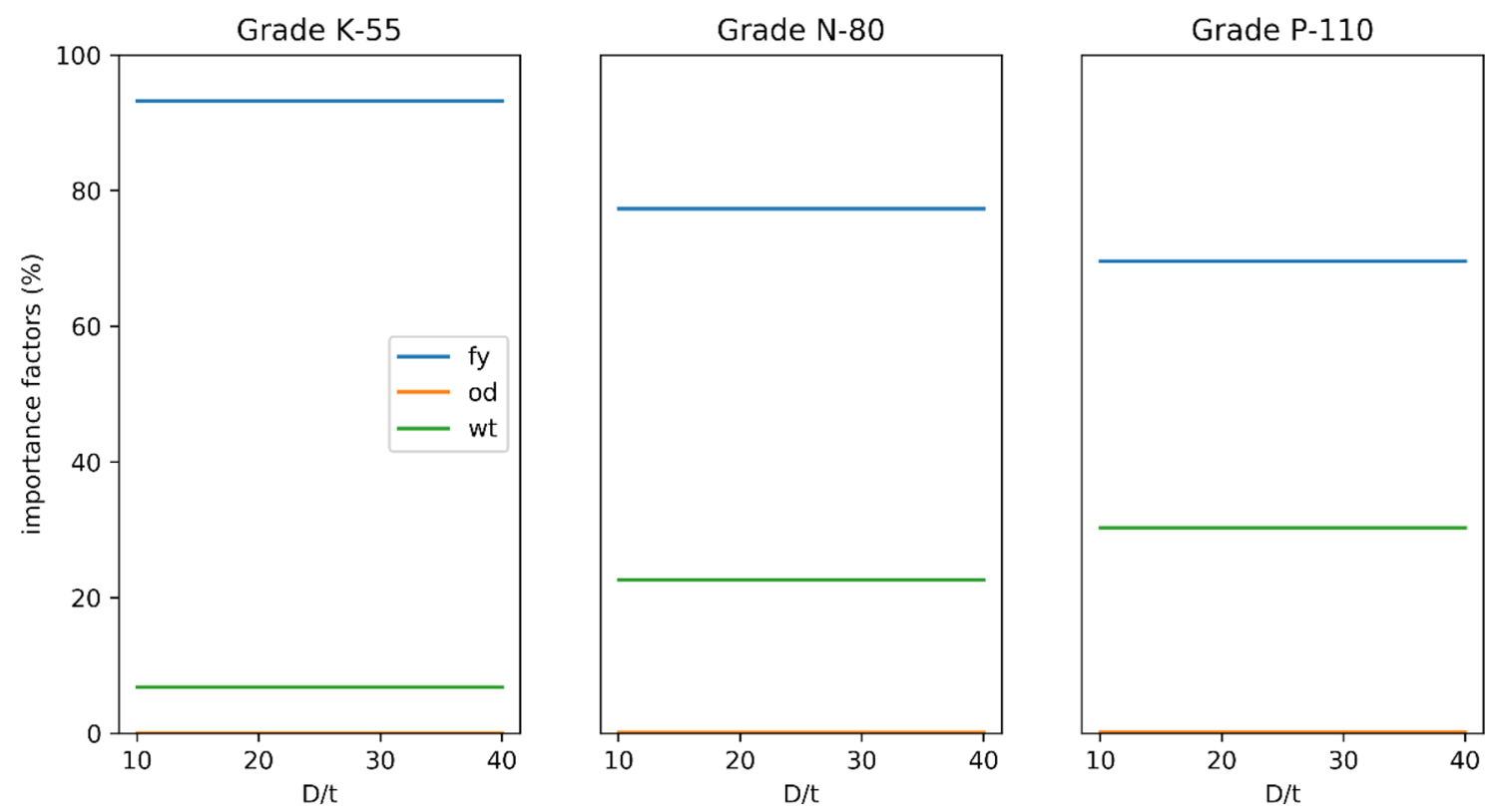

Fig. 8 Importance factors over D/t range for burst analysis
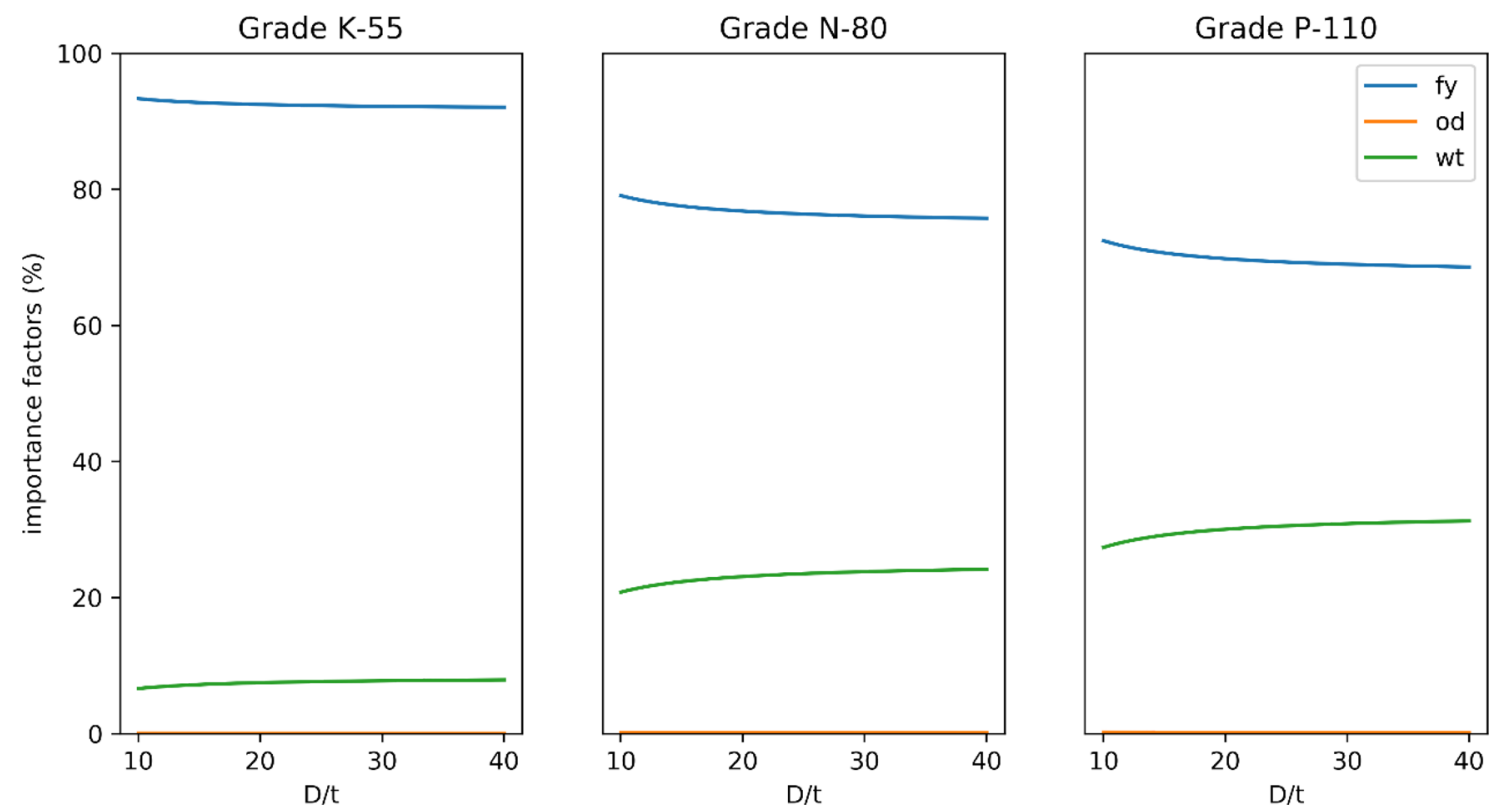

Fig. 9 Importance factors over D/t range for axial force analysis

Observing Fig. 10, when only internal pressure, external pressure or axial force is applied separately, the result converges with the probabilities presented in the previous section. In the case of reduced collapse, i.e., negative pressure differential with positive axial force, increasing of tension reduces $P_{\mathrm{f}}$ in all cases. In an overview, the values of the probabilities of exceedance vary significantly over grades, but only vary over $D / t$ in collapse region. This may be an indicative of how minimum safety factors can be calibrated for a serviceability limit state, where high probabilities of exceedance should lead to higher values of minimum safety factors. Considering this, variable minimum safety factors may be defined for different casing tubulars in order to achieve constant safety probabilities. For a more complete evaluation, von Mises limit state is also verified in the same guidelines, as follows. 

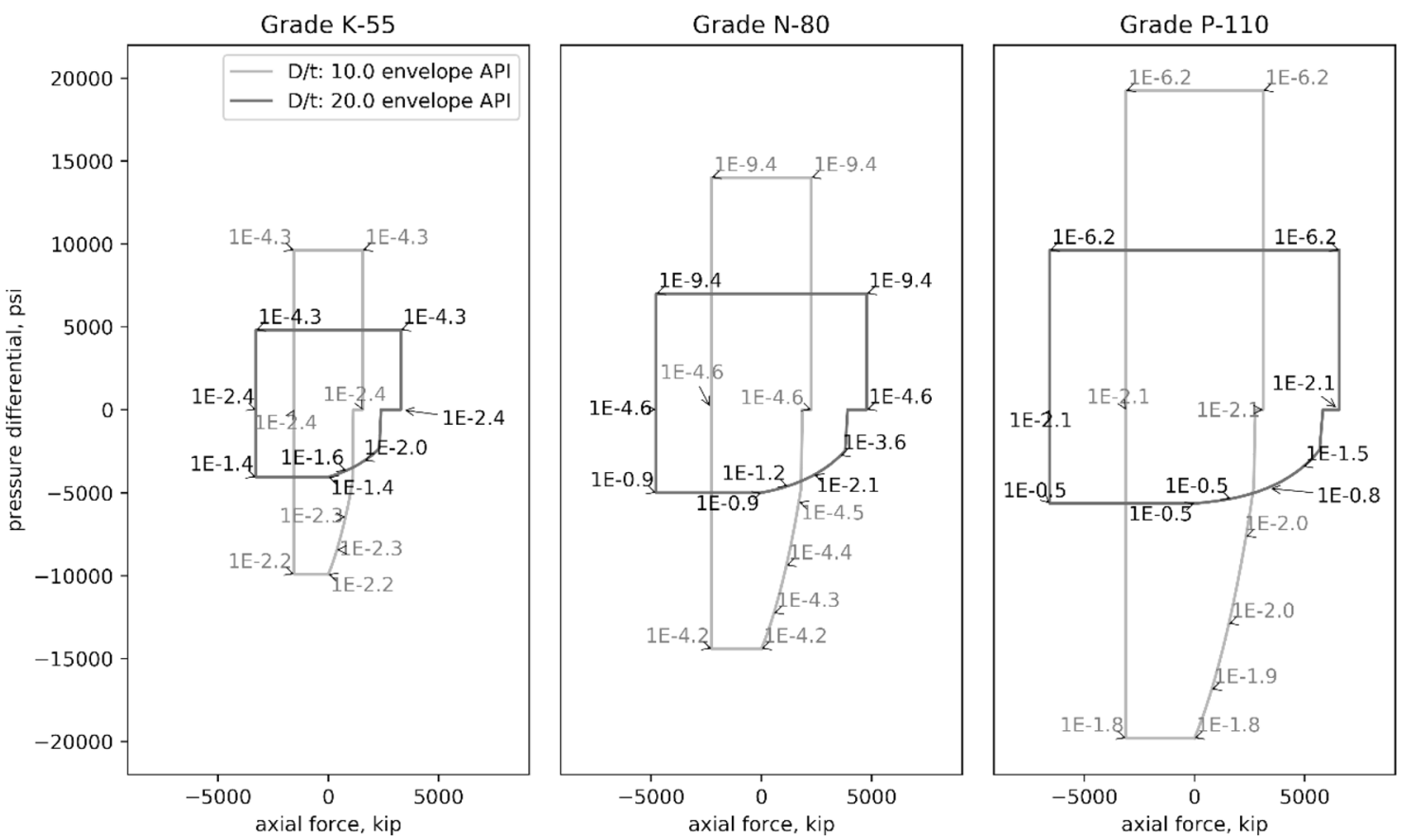

Fig. 10 Probabilities of exceeding SLS at API design envelope for grades K55, N80 and P110, with $\boldsymbol{D} / \boldsymbol{t} 10.0$ and 20.0

Reliability applied for von Mises limit state refers to the probability of equivalent stress $\sigma_{e}$ (Eq. 16) exceeds steel yield stress $Y_{p}$. Thus, the limit state equation may be written as
$G\left(Y_{p}, D, t\right)=Y_{p}-\sigma_{e}(D, t)$

where $\sigma_{e}$ depends on the tube geometry, internal and external pressures, and axial force. In Fig. 11 the probabilities of
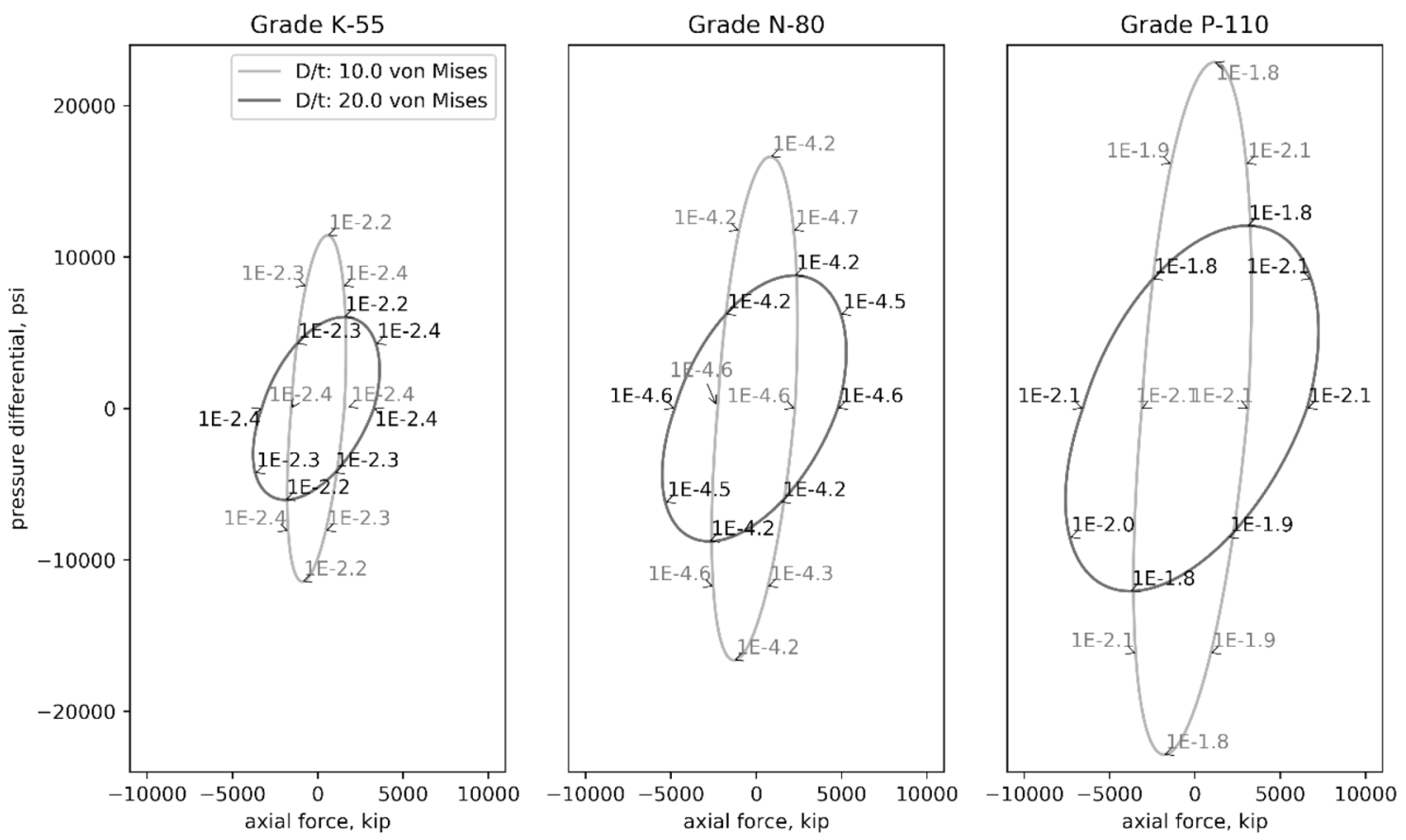

Fig. 11 Probabilities of exceeding SLS at von Mises ellipse limit state for grades K55, N80 and P110, with $\boldsymbol{D} / \boldsymbol{t} 10.0$ and 20.0 
failure for von Mises limit state are presented for the load points over the design ellipse.

Regarding Fig. 11, a near constant $P_{\mathrm{f}}$ value is observed for each grade, but this value varies significantly from grades K55 and P110 to grade N80. It may indicate that if a target $P_{\mathrm{f}}$ similar to grades K55 and P110 is desirable, the ellipse for grade N80 could expand proportionally until achieve this target. It is important to remark that the high values observed are associated with the violation of a serviceability condition, and not representative of a severe structural failure.

\section{Typical design scenario}

In a casing design routine, the tubes are selected considering different loading conditions throughout the well depth, along its lifetime. Depending on the geopressure conditions, some usual and extreme scenarios may occur along the drilling, casing, completion and production stages. These kinds of scenarios have to be predicted and simulated in the well design. A so-called displacement to gas load case is defined when a gas invades the drilling column, increasing drastically the expected internal pressure levels on the casing system, leading to drastic accidents as a blowout. For design purposes, it is considered that the last $2 / 3$ of well depth are occupied by gas.

It is assumed a drilling of a $5700 \mathrm{~m}$ depth well, under $2000 \mathrm{~m}$ of water depth. The calculations of each pressure term are neglected, for the sake of conciseness. The loading profile is shown in Fig. 12, which defines the differential pressure over the depth, resulting in a burst (internal pressure) failure mode overall. This analysis assumed a $103 / 4 \mathrm{in} 85.3 \mathrm{lb} / \mathrm{ft}$ tube, which is widely applied as intermediate casing string, presenting a wall thickness of $0.797 \mathrm{in.} \mathrm{The} \mathrm{three} \mathrm{grades} \mathrm{K55,} \mathrm{N80} \mathrm{and} \mathrm{P110}$ are adopted, to verify safety levels involved.

A deterministic design may select grade P-110, since the load is totally inside of its von Mises and API envelopes, as shown in Fig. 13.

The reliability evaluations are done every $100 \mathrm{~m}$. As internal pressure is greater than external pressure along all well depth, reliability is applied for API burst and von Mises limit states. The results are shown in Fig. 12, in which the failure probability values are log scaled. In addition, the corresponding safety factors (SF), applied in the design practice, are presented in a comparative analysis. $\mathrm{SF}$ is defined as the ratio between the tube strength value and the corresponding applied load.

As expected, the grade K55 tube reaches higher failure probability values. The severe values of pressure from $2000 \mathrm{~m}$ up to $4000 \mathrm{~m}$ lead to unallowable levels of $P_{f}$ for this tube, confirming its inadequacy for this scenario.

Considering that the differential pressure is constant up to around $3223 \mathrm{~m}$, which considered the fluid inflow, the burst failure probabilities remain unchanged. From this point on, until $5700 \mathrm{~m}$, it is observed a quasi-linear decrease in $P_{f}$ values, referring to the reduction in applied pressure, due to the low specific weight of the invading fluid.
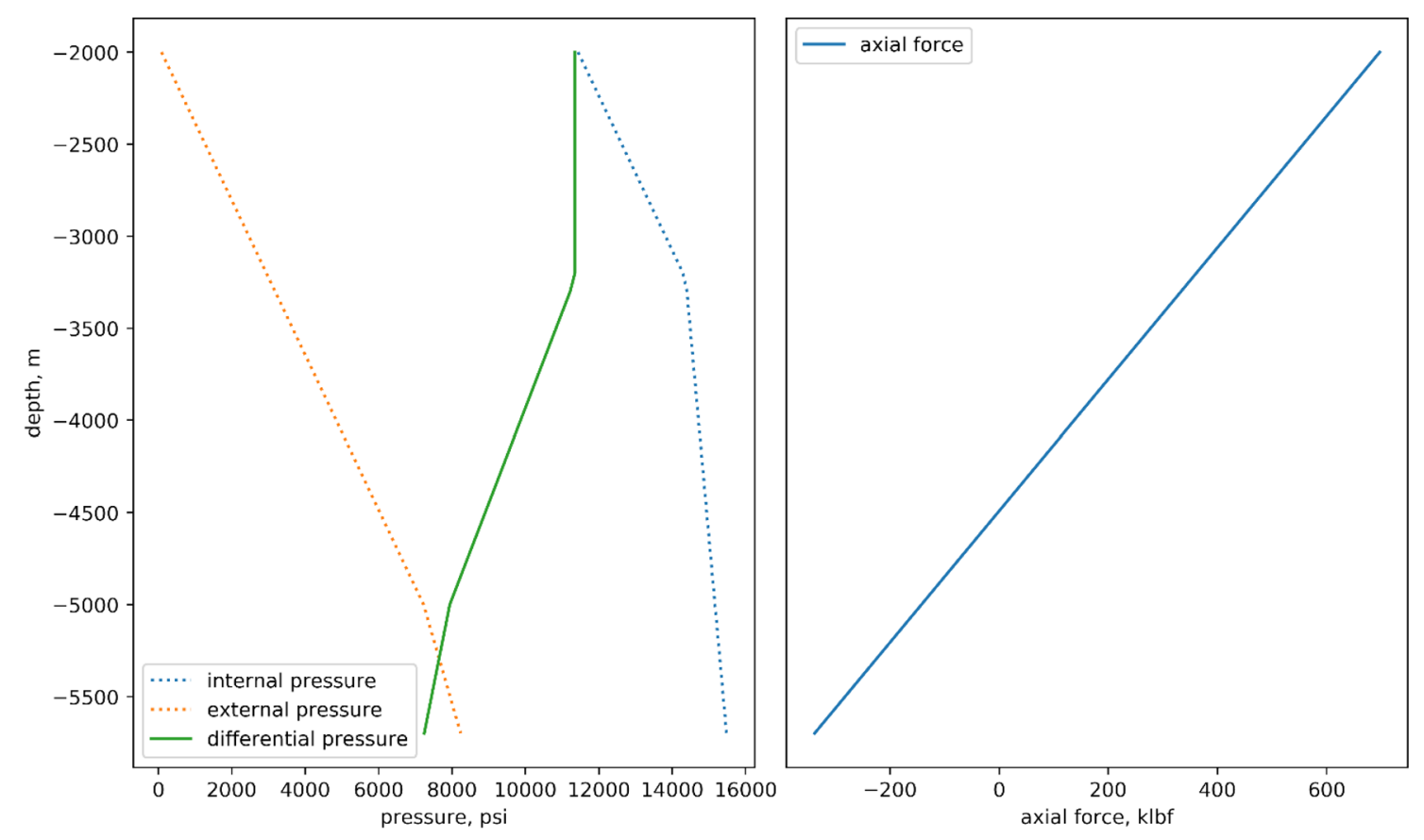

Fig. 12 Loading profile on the adopted scenario 
Fig. 13 von Mises and API envelopes for K55, N80 and P110 grades with $D=10.75$ " and $t=0.797$ ". The loads for displacement to gas scenario are also displayed

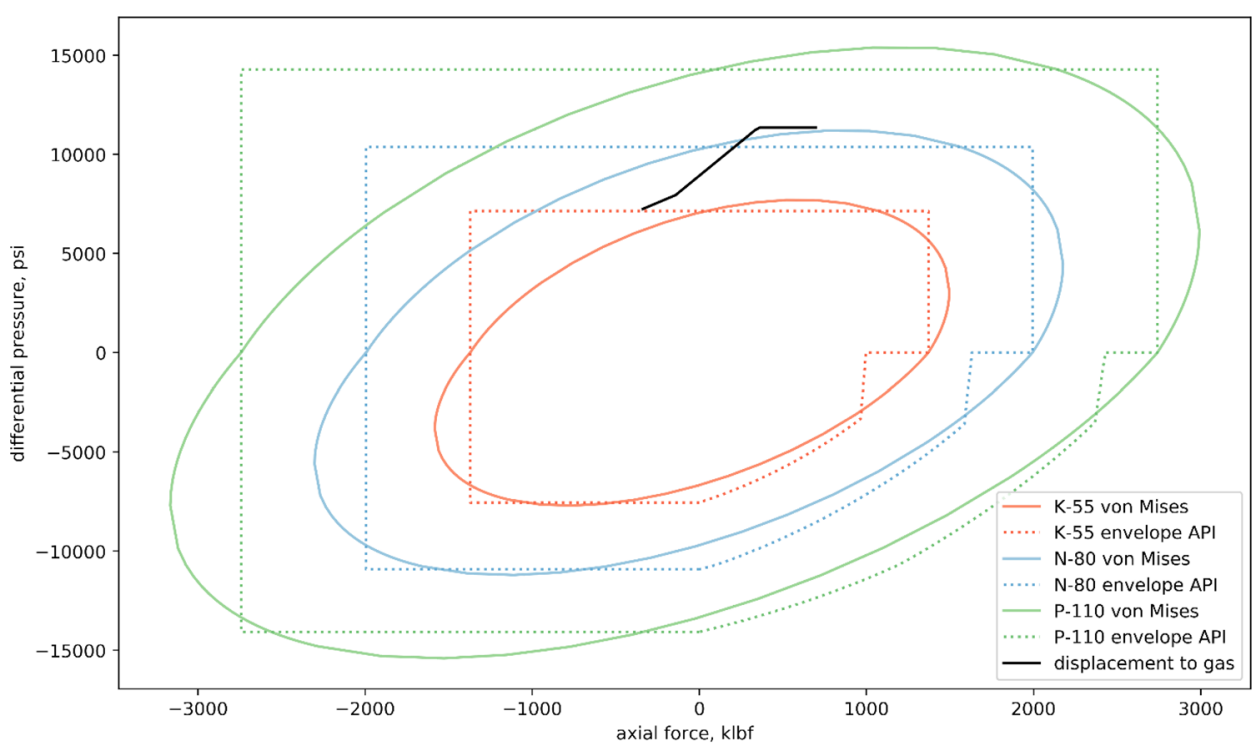

Fig. 14 Probabilities of exceeding SLS along the depth of the well for the adopted scenario

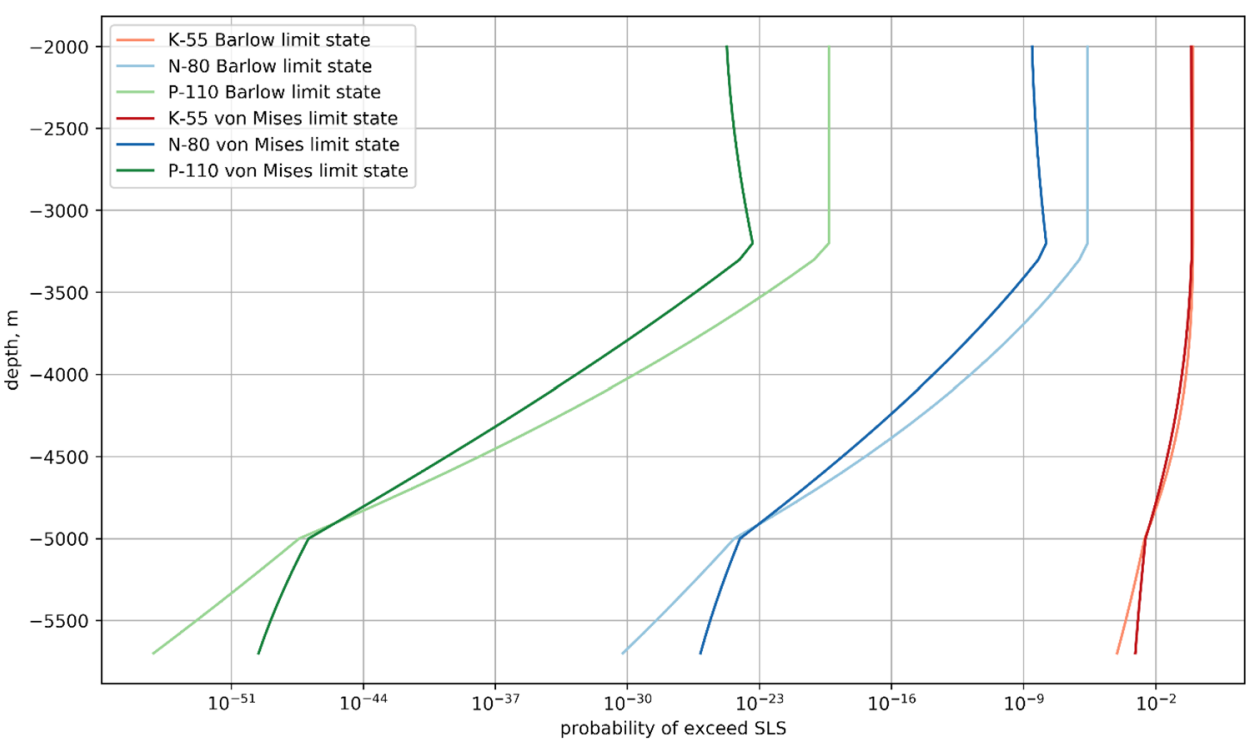

In Fig. 14 Barlow limit state achieves greater values of $P_{f}$ when axial force is positive (tension), but von Mises limit state overtakes when axial force becomes negative (compression). It is recommended to use the critical value in any depth. At surface, grade N80 reaches its maximum value of $P_{\mathrm{f}}$ about $10^{-7.8}$. This value is low even for a SLS, even though the deterministic design would not select this grade. It is also noticed that the probabilistic evaluation seems to be more sensitive to axial changes rather the usual safety factors deterministic evaluation.

This kind of analysis allows comparing the $P_{\mathrm{f}}$ values in any point along the well depth with the minimum safety factors usually employed in casing design. This discussion is not developed here, considering that each oil company defines these factors.

\section{Conclusions}

The classical collapse, burst and axial strength models are revisited in the light of a probabilistic approach, besides the triaxial von Mises criterion. Moreover, the reliability analysis is applied to the verification of a displacement to gas load scenario.

Regarding the analysis on the collapse design formulation, it is noticed that there are some high failure probability values associated with the collapse design strength, when a deterministic load equals to the minimum casing strength is considered. Moreover, the non-uniformity of the safety levels across the slenderness $D / t$ is not a proper behavior, for structural design purposes. The importance 
factors values indicate that wall thickness is the most influent random variable in the achieved failure probability. It stands out the need for application of more robust formulations, which describe more accurately the collapse mechanism of tubes, as Klever-Tamano equation.

For burst analysis, moderate failure probability values are verified. The importance factor values indicate that the material yield limit is the most influent random variable in the achieved failure probability, followed by the wall thickness. The supposed conservative nature of the burst equation, posed by several authors in the literature, is apparently verified here.

The evaluation on the axial strength model reveals some high values of $P_{\mathrm{f}}$, specially associated with P110 steel grade. The yield strength stands out as the dominant random variable, also followed by the wall thickness. There is a slight variation of the importance factors across $D / t$, in all the grades adopted.

There is an aspect that should be emphasized at this point, relative to some concerning values of $P_{f}$ obtained in the above-mentioned models: the statistical database (Table 1) reflects manufacture and inspection systems from 1977 to the 2000s and then is expected that the products manufactured by using modern technologies perform better, in terms of a lower dispersion in dimensional and mechanical parameters.

The application of a probabilistic evaluation in the casing design practice can be done by procedures such as the scenario analysis presented. It brings robustness to the analysis and aids the designer in decision-taking processes aiming both investment savings in simple wells and feasibility in complex wells. FORM is suggested to be applied in these analyses due to its good accuracy with Monte Carlo results for $P_{\mathrm{f}}$ greater than $\sim 10^{-4.4}$, since it is evaluated much faster than Monte Carlo. This also should allow fast integrity verifications in casing design and real-time operation activities. Increasing the complexity of the FORM for a second-order method (SORM) may not improve the results significantly since the agreement of FORM with Monte Carlo is already reasonable.

In this context, the need of detailed analysis both on casing design formulation and about the non-deterministic nature of strength parameters stands out. The standardization codes and oil/gas companies are interested in these issues since the last two decades, and some scientific and technical publications have been developed. Some effort has also to be done in order to consider combined load cases in a probabilistic approach, focusing on the stochastic behavior of environmental load scenario. Reliability techniques can support industry and designers, identifying aspects in which the manufacturing process must be improved in its accuracy and quality inspection. It must be noted that in service conditions the tubes may ovalize due to high pressures and worn out due to corrosion and friction of drill pipes and other equipment, where these damages change the tube geometry and may decrease its strength significantly. More robust strength formulations or numerical models that consider these effects will allow probabilistic methods to be applied.

It should be remarked that the results presented herein are only indicatives of the probabilistic behavior associated with the design formulations studied. The $P_{\mathrm{f}}$ values must be interpreted with prudence, as they refer to a specific database, provided by the code ISO 10400 (2007).

Finally, it is important to remind that the probabilities calculated generally refer to the exceedance of serviceability limit states, and not to rupture, so that the tubes should present a remaining strength after this point. Moreover, in design practice, the minimum safety factors defined by the companies overall are usually superior to 1.0 .

Funding This work was supported by Petróleo Brasileiro S.A. (Petrobras).

Open Access This article is licensed under a Creative Commons Attribution 4.0 International License, which permits use, sharing, adaptation, distribution and reproduction in any medium or format, as long as you give appropriate credit to the original author(s) and the source, provide a link to the Creative Commons licence, and indicate if changes were made. The images or other third party material in this article are included in the article's Creative Commons licence, unless indicated otherwise in a credit line to the material. If material is not included in the article's Creative Commons licence and your intended use is not permitted by statutory regulation or exceeds the permitted use, you will need to obtain permission directly from the copyright holder. To view a copy of this licence, visit http://creativecommons.org/licenses/by/4.0/.

\section{References}

Abbassian F, Parfitt SHL (1995) Collapse and post collapse behavior of tubulars: a simple approach. In Proceedings of SPE production operations symposium

Adams AJ, Grundy KC, Kelly CM, Lin B, Moore PW (2018) The Barlow equation for tubular burst: a muddled history. In Proceedings of IADC/SPE drilling conference and exhibition

American Petroleum Institute API (1999) API Bull 5C3: Bulletin on formulas and calculations for casing, tubing, drill pipe and line pipe properties. Bulletin

American Petroleum Institute-API (2008) API TR 5C3: Technical report on equations and calculations for casing, tubing, and line pipe used as casing or tubing; and performance properties tables for casing and tubing. Technical report

American Petroleum Institute-API (2010) API 5CT: Specification for casing and tubing. Specification

Ang A, Tang W (2007) Probability concepts in engineering: emphasis on applications in civil \& environmental engineering. Wiley, New York

Breitung K (1984) Asymptotic approximations for multinormal integrals. J. of Eng. Mech. Div. 110:357-366

Burres CV, Tallin AG, Cernocky EP (1998) Determination of casing and tubing burst and collapse design factors to achieve target levels of risk, including influence of mill source. In Proceedings of

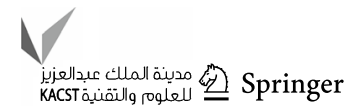


SPE applied technology workshop on risk based design of well casing and tubing

Ditlevsen O, Madsen HO (2005) Structural reliability methods. Technical University of Denmark, Denmark

Gao J, Shi W, Cui S, Jiao Y (2019) Reliability analysis for seismic capacity of casing strings. J Pet Explor Prod Technol (online preprint). https://doi.org/10.1007/s13202-019-00765-8

Hasofer A, Lind N (1974) An exact and invariant first order reliability format. J. Eng. Mech. Div. 100:111-121

Huang W, Gao D (2015) A theoretical study of the critical external pressure for casing collapse. J Nat Gas Sci Eng 27:290-297

International Organization for Standardization-ISO (2007) ISO TR 10400: Petroleum, petrochemical and natural gas industriesEquations and calculations for the properties of casing, tubing, drill pipe and line pipe used as casing or tubing. Technical report

Joint Committee on Structural Safety-JCSS (2002) Probabilistic model code

Ju GT (1998) A reliability approach to the design of OCTG tubulars against collapse. In Proceedings of SPE applied technology workshop on risk based design of well casing and tubing

Klever F (2010) Formulas for rupture, necking, and wrinkling of oil country tubular goods under combined loads. SPE Journal 15:834-855

Klever F, Stewart G (1998) Analytical burst strength prediction of OCTG with and without defects. In Proceedings of SPE applied technology workshop on risk based design of well casing and tubing

Klever F, Tamano T (2006) A new OCTG strength equation for collapse under combined loads. SPE Drill Complet 21:164-179

Liao H, Guan Z, Long G (2012) Quantitative risk assessment on safety and reliability of casing strength for oil and gas wells. Energy Procedia 17:429-435
Adams AJ, Warren AVR, Masson, PC (1998) On the development of reliability-based design rules for casing collapse. In proceedings of spe applied technology workshop on risk based design of well casing and tubing

Melchers RE, Beck AT (2017) Structural reliability analysis and prediction, 3rd edn. Wiley, Hoboken

Mohammed AI, Oyeneyin B, Atchison B, Njuguna J (2019) Casing structural integrity and failure modes in a range of well typesa review. J Nat Gas Sci Eng. https://doi.org/10.1016/j.jngse .2019 .05 .011

Nataf MA (1962) Détermination des distributions de probabilités dont les marges sont données. Comptes Rendus de l'Académie des Sciences 225:42-43 (in French)

Rackwitz R, Fiessler B (1978) Structural reliability under combined random load sequences. Comput Struct 9:489-494

Rahman SS, Chilingarian GV (1995) Casing design-theory and practice, vol 42. Developments in petroleum science. Elsevier, Amsterdam, p 372

Tamano T, Mimaki T, Yanaimoto S (1985) A new empirical formula for collapse resistance of commercial casing. Nippon Steel Tech. Report 26:19-26

von Mises R (1913) Mechanik der festen Körper im plastisch deformablen Zustand. Math. Phys. 1:582-592 (in German)

Publisher's Note Springer Nature remains neutral with regard to jurisdictional claims in published maps and institutional affiliations. 